\title{
Effects of Progressive Increased Intensity of PNF Stretching on Hamstring Force and Flexibility
}

\author{
Wootaek Lim ${ }^{1,2}$ \\ ${ }^{1}$ College of Health and Welfare, Woosong University, Republic of Korea \\ ${ }^{2}$ Woosong Institute of Rehabilitation Science, Woosong University, Republic of Korea
}

Received July 21, 2020; Revised August 26, 2020; Accepted September 29, 2020

\section{Cite This Paper in the following Citation Styles}

(a): [1] Wootaek Lim, "Effects of Progressive Increased Intensity of PNF Stretching on Hamstring Force and Flexibility," International Journal of Human Movement and Sports Sciences, Vol. 8, No. 5, pp. 186 - 192, 2020. DOI: 10.13189/saj.2020.080505.

(b): Wootaek Lim (2020). Effects of Progressive Increased Intensity of PNF Stretching on Hamstring Force and Flexibility. International Journal of Human Movement and Sports Sciences, 8(5), 186 - 192. DOI: 10.13189/saj.2020.080505.

Copyright $\bigcirc 2020$ by authors, all rights reserved. Authors agree that this article remains permanently open access under the terms of the Creative Commons Attribution License 4.0 International License

\begin{abstract}
In most previous studies of PNF stretching performed at different intensities, the subjects were provided with the intensities in a randomized order. In addition, subjects were aided in maintaining a steady level of contraction force using a dynamometer as visual feedback. However, such a procedure differs from the procedure used in actual clinical settings. This study aimed to examine the effect of the applied order of three different intensities of PNF stretching on the ability to regulate the contraction force and increase muscle flexibility. During the $1^{\text {st }}$ period, three different stretching intensities were applied in the order of $25 \%, 50 \%$, and $75 \%$ of MVC in the group A and in the order of $75 \%, 50 \%$, and $25 \%$ of $\mathrm{MVC}$ in the group $\mathrm{B}$. This order was reversed during the $2^{\text {nd }}$ period. The contraction force was measured using the strain gauge and hamstring flexibility was measured using active knee extension. There was no significant difference between target and contraction intensity at three target intensities in both groups. Additionally, contraction forces between different intensities clearly were distinguished by subjects in both groups. In Group A, increase in flexibility was significantly higher in the $1^{\text {st }}$ period than in the $2^{\text {nd }}$ period. Healthy adults could clearly differentiate and regulate contraction intensity when the intensities were differentially applied at $25 \%$ intervals. The higher target intensity compliance and increase in flexibility may be anticipated when progressively increase intensity was applied, compared to the same factors in reverse order.
\end{abstract}

Keywords Hamstring Muscles, Isometric Contraction,
Proprioceptive Neuromuscular Facilitation Stretching, Range of Motion

\section{Introduction}

The mechanism behind the effects of proprioceptive neuromuscular facilitation (PNF) stretching on muscle flexibility still remains partly unclear; however, an increase in flexibility has been clearly verified [1]. During stretching, numerous factors may influence the quantitative increase in flexibility; among them, the intensity, in particular, is considered a key factor [2]. Previous studies examining the effects of the different intensities of stretching on flexibility confirmed that moderate-intensity or above stretching leads to a significant increase in flexibility; moreover, low-intensity stretching also sometimes leads to an increase in flexibility [3-5]. Nevertheless, previous studies conducted in laboratory conditions have several limitations when it comes to the clinical application of their findings.

First, the order of different intensities poses a limitation. In previous studies where more than two different intensities were used, the method of applying the intensity could be categorized into two types: the stretching may be performed in a randomized order for all intensities within a defined subject $[4,6-8]$ or with only one intensity randomly assigned to each subject $[3,5,9]$. The former method 
applies randomized order such that the order of different intensities on flexibility cannot be analyzed. In the latter method, the stretching is performed at a single intensity so that the characteristics of individual subjects may easily influence the results and, as in the former method, the effects of the order of different intensities on flexibility cannot be determined. In the preliminary study, when PNF stretching at various intensities was provided with either a progressive increase or decrease in intensity, the perceived exertion was found to vary. Unlike in the research setting, in clinical settings progressively increasing intensity is applied rather than a randomized order of intensity. In addition, even for resistance exercises that use isometric contraction as in the case of PNF stretching, progressively increasing intensity is widely used $[10,11]$.

Second, visual feedback is different in clinical settings. In most previous studies, the contraction force being measured in the experiment was provided to the subjects in real time to aid them in self-regulation of the muscle contraction according to the target intensity $[3,5,9,12]$. The subjects in such cases were able to monitor the contraction force as a numerical value so that it is possible to perform a muscle contraction of the desired level based on a clear differentiation between two or more intensities assigned for the experiment. However, in clinical or sports settings, to measure the muscle force and provide data to the subjects in real time is difficult. For the measurement of muscular strength, an isokinetic dynamometer (e.g., Biodex, Cybex) is the gold standard, but the device is too large for clinical application and its skilled use demands considerable training, limiting its application [13]. In practice, the intensity is generally adjusted based on both the subjective experience of the physical therapist or trainer and the pain response of the subjects as observed during PNF stretching. In other words, without visual feedback from the device specialized for measurement, it may be challenging for subjects to clearly differentiate between the intensities suggested by the practitioner during performance.

The purpose of this study was to examine 1) whether the subjects could clearly differentiate between three intensities when performing muscle contraction; 2) how close the contraction intensity is to the target intensity; and 3 ) whether there is a difference in the increase in muscle flexibility upon varying the order of intensity, when the subjects were provided with three different intensities in two orders. In contrast to previous studies, visual feedback was not presented to allow subjects to perform PNF stretching in an environment similar to the clinical setting.

\section{Materials and Methods}

\subsection{Subjects}

A total of 28 subjects (age $22.1 \pm 1.6$ years, height
$165.6 \pm 8.3 \mathrm{~cm}$, weight of $62.2 \pm 12.0 \mathrm{~kg}$ ) participated in the study and were randomly divided into two groups (Figure 1). The participants in this study had hamstring tightness (defined as active knee extension test $>20^{\circ}$ ). Individuals who had undergone surgery to the hip, knee, or ankle joint within the past 6 months, or active pain were excluded. The study was approved by the institutional review board. Informed consent was obtained from each participant.

\subsection{Procedures}

Each subject was instructed to lie on the treatment table in the supine position. The hamstring flexibility in the randomly selected lower extremity was measured using the active knee extension (AKE) test. The subject was instructed to perform $90^{\circ}$ flexion of the hip and knee joint for the AKE test. While the subject slowly performed knee extension, the examiner recorded the knee extension angle upon maximal knee extension. The Pre-AKE was calculated as 180-knee extension angle. After measuring the Pre-AKE, PNF stretching was performed. First, the subject performed the straight leg raise, while the leg was slowly and passively lifted by the examiner using a sling system to the point right before discomfort or pain. The ankle joint of the subject was immobilized using the strap connected to one side of the sling wire, while the other side of the sling wire was fixed to the ceiling. Here, the position of the sling system was adjusted such that the angle between the ceiling and leg was $90^{\circ}$. The contraction forces were measured by a strain gauge (Re-live Inc., Kimhae, Korea). At maximal hip extension, three trials of maximal voluntary contraction (MVC) were carried out; during the MVC, the examiner used verbal encouragement to help the subject achieve the maximal force. Once the muscle had contracted, it was maintained for 5 seconds per trial. There were 10 -second rest periods between trials. During isometric contraction, the examiner controlled the horizontal movement of the subject's leg on both sides of the ankle joint to prevent the leg from swinging sideways. After three MVC trials, an additional set of three different submaximal contractions was performed after 3 min rest. For Group A, the target intensity was defined in the order of $25 \%, 50 \%$, and $75 \%$ of MVC during the $1^{\text {st }}$ period. For Group B, the target intensity was defined in the order of $75 \%, 50 \%$, and $25 \%$ of MVC during the $1^{\text {st }}$ period. As in the case of MVC, three trials were carried out for each level of submaximal contraction, after which the mean of the three values was used. The contraction force measured at each intensity was divided by the maximal contraction forces measured during MVC, and multiplied by 100 to calculate the contraction intensity (\% MVC). After the completion of the $1^{\text {st }}$ period of submaximal contractions at three different intensities, the AKE test was performed, and the measured values were recorded as Post-AKE. The $\triangle \mathrm{AKE}$ was calculated as Post-AKE - Pre-AKE. After the $1^{\text {st }}$ period, the subject was given 10 min to rest. During the 
$2^{\text {nd }}$ period, an identical process was followed using the other lower extremity. In the $2^{\text {nd }}$ period, however, the three different intensities were provided in the opposite order to that in the $1^{\text {st }}$ period.

\subsection{Data Analysis}

Normality of data was assessed using the Shapiro-Wilk test. The one sample Wilcoxon test was used to analyze the difference between target and contraction intensity. The Mann-Whitney $U$ test was used for the analysis of the difference in contraction force between Groups A and B. The Wilcoxon Signed-Rank test was used to evaluate each group between the $1^{\text {st }}$ and $2^{\text {nd }}$ periods. In addition, the Friedman test was used along with Wilcoxon Signed-Rank test post hoc analysis to examine the difference in contraction forces measured at three different target intensities. Spearman Rank Correlation was used for analysis of the relationship between 'target intensity' and 'the difference between target and contraction intensity'. Carryover effect was assessed using the Mann-Whitney U test. IBM SPSS Statistics 25 (IBM Corp., Armonk, NY, USA) was used for analysis of data and statistical significance was set at $p<0.05$, except for the data analyzed by the Wilcoxon Signed-Rank test after the Friedman test (set at $\mathrm{p}<0.05 / 6$ ). The Wilcoxon Signed-Rank test was used to compare increases in flexibility before and after isometric contractions in each group during the $1^{\text {st }}$ and $2^{\text {nd }}$ periods, and to compare the difference between the $1^{\text {st }}$ and $2^{\text {nd }}$ periods in each group. The Mann-Whitney U test was used for the analysis of the difference in hamstring flexibility between group A and B. All values were reported as mean \pm standard deviation.

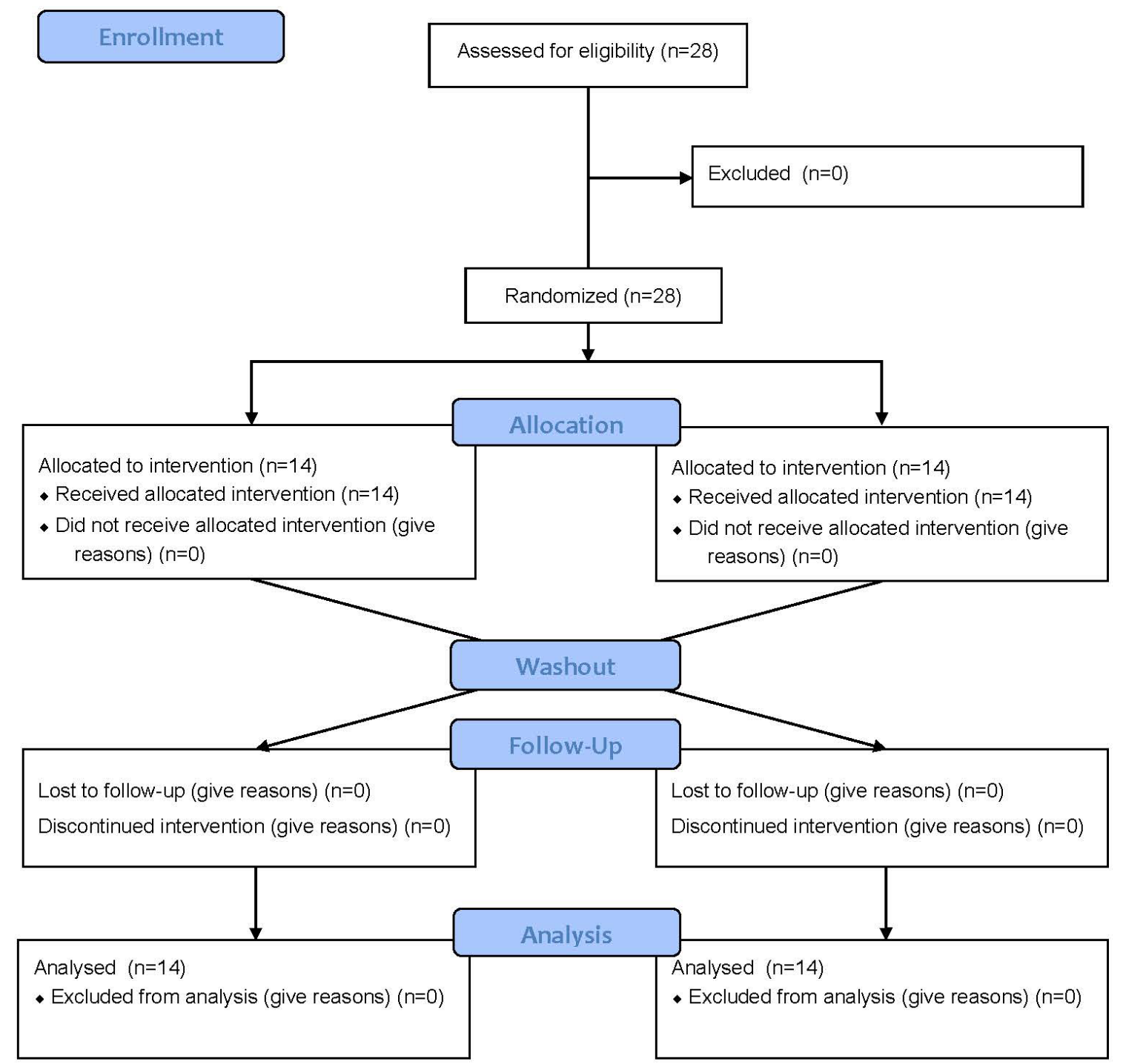

Figure 1. CONSORT flow diagram 


\section{Results}

There was no significant difference between target and contraction intensity at $75 \%(\mathrm{p}=0.650,0.775), \quad 50 \%$ $(p=0.691,0.955)$, or $25 \%$ of MVC $(p=0.334,0.865)$ in Group A during the $1^{\text {st }}$ period and $2^{\text {nd }}$ period (Figure 2a) and at $75 \%(\mathrm{p}=0.394,0.078), 50 \%(\mathrm{p}=0.910,0.910)$, and $25 \%$ of MVC ( $\mathrm{p}=0.532,0.496)$ in Group B during the $1^{\text {st }}$ period and $2^{\text {nd }}$ periods (Figure $2 b$ ). The mean contraction intensity between Groups A and B at each target intensity was not significantly different. In Group B, a significant relationship $(\mathrm{p}=0.044)$ between target intensity and difference between target and contraction intensity was observed during the $1^{\text {st }}$ period (Figure 3 ).
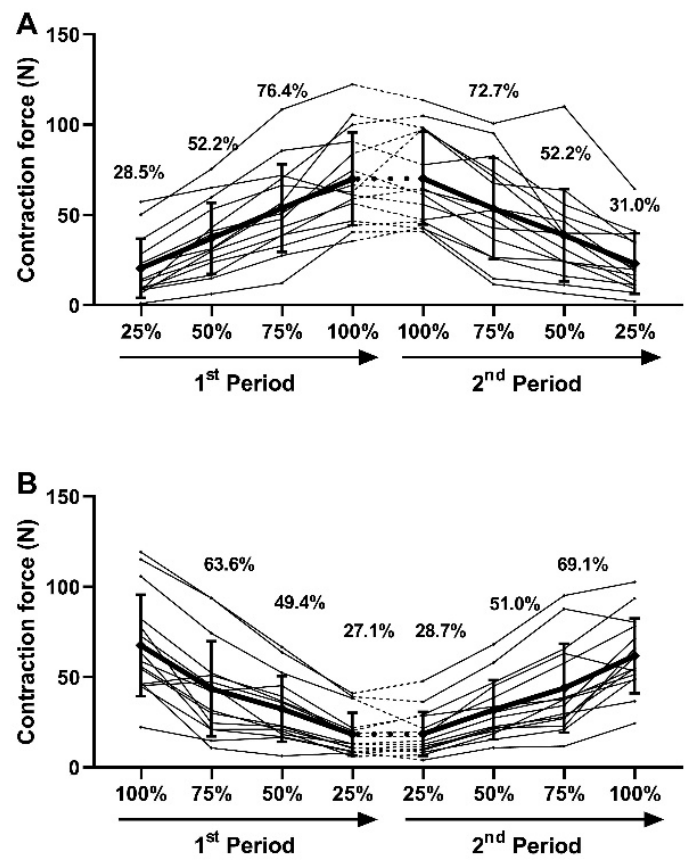

Figure 2. Changes in contraction force during $1^{\text {st }}$ and $2^{\text {nd }}$ period in Group A (A) and Group B (B)
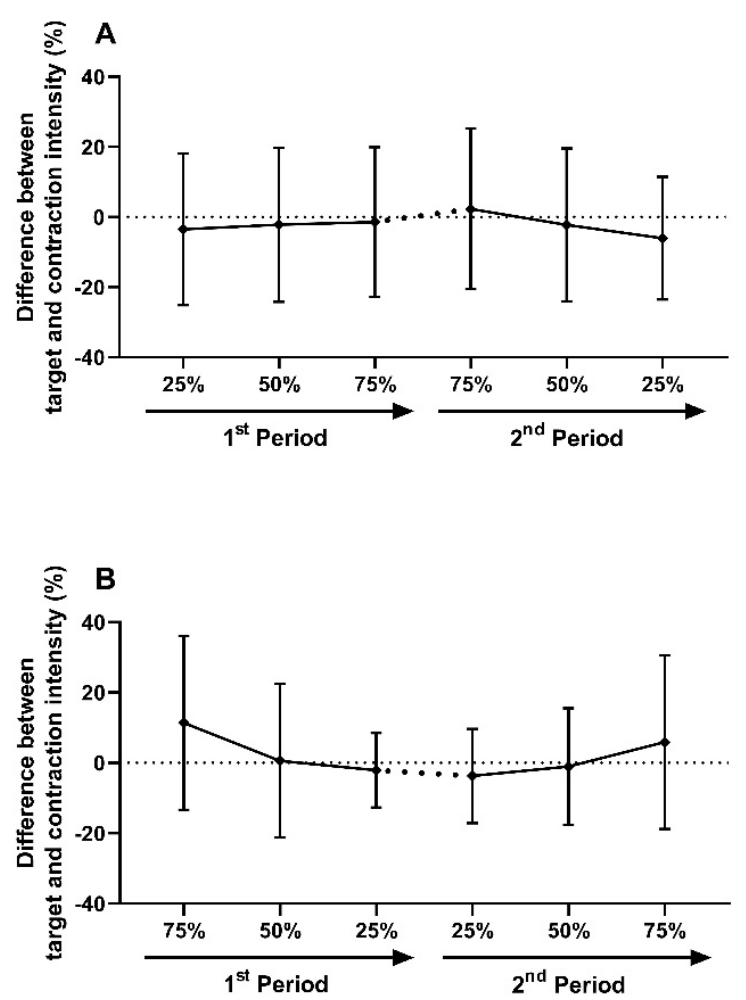

Figure 3. Difference between target and contraction intensity in Group A (A) and Group B (B)

In the within-group comparison for each period, contraction forces between $100 \%$ and $75 \%, 100 \%$ and $50 \%, 100 \%$ and $25 \%$, and $75 \%$ and $25 \%$ of MVC could be distinguished in Group A and Group B. No carryover effect on contraction force was found.

Hamstring flexibility was significantly increased after submaximal isometric contractions during each period in both groups $\mathrm{A}$ and $\mathrm{B}$. In Group $\mathrm{A}, \triangle \mathrm{AKE}$ was significantly higher in the $1^{\text {st }}$ period than in the $2^{\text {nd }}$ period $(p=0.017)$; however, there was no difference in the increase in flexibility between the two groups (Figure 4). 


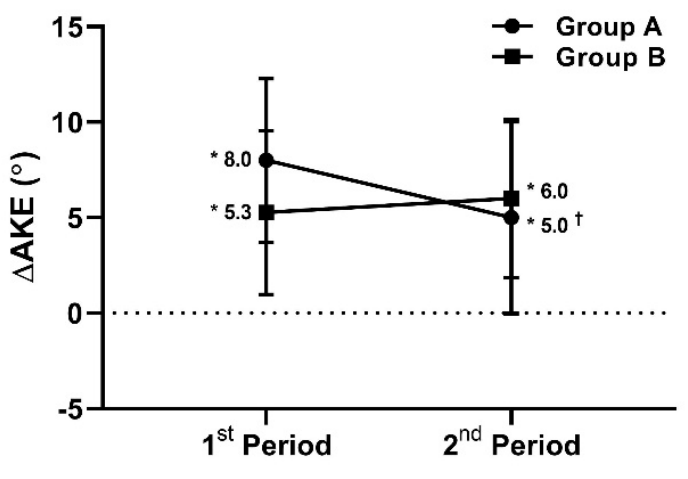

Figure 4. Comparison of increase in hamstring flexibility at $1^{\text {st }}$ period and $2^{\text {nd }}$ period

\section{Discussion}

In this study, similar to conditions in clinical and field settings, information regarding hip extension force was not provided to subjects during voluntary isometric contraction. Three different target intensities were differentially applied at $25 \%$ intervals. The contraction force measured at each target intensity was found to be statistically different from one another. Furthermore, there was no significant difference between contraction intensity (normalized to MVC) and target intensity. This implied that healthy young adults could regulate the level of muscle contraction by clearly differentiating intensities without visual feedback, as well as being able to perform muscle contraction of a desired level based on the intensity imposed by another individual. Much of the information obtained during muscle contraction is sent to the central nervous system in real time through afferent pathways, based on which the human body can perform detailed and accurate movements [14-17]. Such feedback mechanisms are realized through proprioception and may be decreased or increased by an external factor [18-20]. The most well-known proprioceptors in the human body are the muscle spindle and Golgi tendon organ (GTO). The muscle spindle is known to provide information regarding muscle length and velocity. Recently, the changes in muscle length, as well as joint position were reported, to exert an influence $[15,21]$. The GTO is known to detect changes in muscle force rather than absolute force, as it provides information regarding muscle force [22]. In addition, the afferent input is sent to the CNS by mechanoreceptors in the glabrous skin and chemo-sensitive receptors (type III and IV) found in the muscle tissue [23-25]. The feedback mechanism provides only information obtained during a movement so that it cannot influence the step prior to the movement. For a detailed and accurate movement to be performed, it is sometimes necessary to influence the step prior to the movement. The feedforward system sends an anticipatory input to the sensory area before a movement is generated; consequently, much recent focus has been placed on the feedforward mechanism. The feedforward mechanism incorporates the concept of corollary discharge (an internal copy of the motor signal transmitted from the motor to sensory areas) [26]. This copy of the signal cannot create a movement on its own, but plays a crucial role in determining sensory discrepancy through comparison with reafferent signals coming from the sensory system $[22,27,28]$. In recent studies, the location where signals arise is not simply restricted to the motor cortex [29]. The integrated information from the feedforward and feedback mechanisms is presumed to assist perceived exertion to approach the actual contraction intensity. Recently, a diversity of factors, including psychological stimuli, in addition to the physical properties described so far, have been shown to exert a complex influence [30].

Although there was no significant difference between the target and actual contraction intensity for all three different intensities, the within-group differences in contraction force between the $1^{\text {st }}$ and $2^{\text {nd }}$ periods revealed a significant trend. To be specific, in Group B, a significant negative correlation $(\mathrm{p}=0.044)$ was observed during the $1^{\text {st }}$ period where progressively decreased intensity was applied. On the contrary, during the $2^{\text {nd }}$ period where progressively increased intensity was applied, no significant correlation was found, indicating that the difference between target and contraction intensities in the $2^{\text {nd }}$ period was maintained at a steady level, with no notable change. This was similarly observed in Group A. To put it another way, when progressively decreasing intensity was applied, the difference between target and contraction intensities showed a gradual fall with decreasing intensity, which suggests the possibility of under-production at high-intensity and over-production at low-intensity. According to a previous study examining various intensities, under-production was frequently observed at high-intensity. In a study by Cooper and Jackson (2003), the knee extensor forces were reported to show under-production at $75 \%$ of maximal perceived effort [31]. In Pinceviro (2003), likewise, the knee extensor torque showed under-production at high perceived exertion levels [12]. The under-production at high-intensity is sometimes explained as a subconscious response to reduce the risk of injury caused by muscle contraction at excessive high-intensity and energy expenditure [12]. However, this assumption fails to explain the over-production at low-intensity. In Kumar (1994), over-production was observed for the power grip at $20 \%$ of MVC [32], while in Sheard (2003), it was observed for the hip flexion at $20 \%$ of MVC [7]. The difference between the target intensity and the actual contraction intensity is more likely to be accounted for as a bias arising from each extremity rather than as a protective mechanism. When the assigned target intensity increases or decreases based on $50 \%$ of MVC, the actual contraction intensity shows a comparatively smaller margin of increase or decrease, thereby exhibiting a trend 
in which it is positioned relatively closer to the center, with consequent over-production at low-intensity and under-production at high-intensity. Most previous studies described thus far reported a small difference between target intensity and contraction intensity for moderate-intensity contraction. In this study, the range of contraction intensity at a target of $50 \%$ of MVC was 49.4 $52.2 \%$. In contrast, the range with a target of $25 \%$ of MVC was $27.1-31.0 \%$, higher than the target intensity, while the range with a target of $75 \%$ of MVC was $63.6-72.7 \%$, generally lower than the target intensity (except Group A during the $1^{\text {st }}$ period).

For PNF stretching, the maximal and/or submaximal voluntary isometric contraction is repeatedly performed to increase flexibility. The voluntary isometric contraction in this study was identical to the PNF stretching procedure, based on which an increase in hamstring flexibility across the subjects could be predicted. In both groups, the hamstring flexibility showed a significant increase after repeated isometric contractions regardless of the order of intensities. Additionally, in the case of Group A, the progressively increased intensity (the $1^{\text {st }}$ period) led to a more significant increase in flexibility than the progressively decreased intensity (the $2^{\text {nd }}$ period). Group B, likewise, showed a slightly higher increase in flexibility during the $2^{\text {nd }}$ period in which a progressive increase had been applied. During PNF stretching for the improvement of flexibility, the GTO plays an important role with the muscle spindle. The stimulation of the muscle spindle caused by stretching leads to muscle contraction to prevent overstretched muscles, so that the stretching can be inhibited [33,34]. In contrast, the GTO inhibits the contraction of stimulated muscles, thereby assisting in stretching $[35,36]$. For PNF stretching, the muscle spindle inhibiting the flexibility increase is reduced, whereas the GTO that helps to increase the flexibility is known to be stimulated [6]. Furthermore, the positive effects of neurological properties on flexibility are known to show an extremely sensitive response at low-intensity in comparison to high-intensity stretching $[1,14]$. In addition, local discomfort may be induced at high-intensity stretching, whereas at moderate-intensity stretching, optimized GTO inhibition may be expected [6]. When high-intensity stretching is applied, the muscle stiffness resists muscle-tendon unit elongation, while the soft tissues exhibit microtears with undesirable pain [37,38]. Although no study has yet examined the flexibility increasing effect based on the order of intensity, as in this study, a previous study reported that low-intensity long-duration stretching may be advantageous in increasing flexibility over high-intensity short-duration stretching [39]. The findings of this study and of previous studies collectively suggest that more effective flexibility increase may be anticipated when a progressive increase in intensity is applied, starting from low-intensity, rather than the reverse order. The subjects are also likely to find this order more comfortable.
For the subjects in this study, muscle contraction could be regulated based on the target intensity, but this may have been possible because of the considerably large differences between consecutive intensities (25\%). Thus, further studies should examine the minimum intensity discernable by the human body, by subdividing the intensity interval across various groups. In addition, the subjects in this study were healthy adults, so the findings cannot be generalized to other subject groups.

Regardless of the applied order of intensity, healthy adults could clearly differentiate and regulate contraction intensity when the given target intensities were at least $25 \%$ different. They could also perform muscle contraction according to the demanded intensity. For changes in the within-group difference, applying a progressively decreased intensity led to a fall in the difference between the target and actual contraction intensities, whereas the reverse order did not lead to a notable change. In other words, by applying a progressively increasing intensity in clinical settings, far higher target intensity compliance may be anticipated. Additionally, a far higher increase in flexibility may be anticipated in such cases, compared to that when using the reverse order.

\section{Acknowledgements}

This research was supported by 2020 Woosong University Academic Research Funding.

\section{REFERENCES}

[1] M.J. Sharman, A.G. Cresswell, S. Riek. Proprioceptive neuromuscular facilitation stretching: mechanisms and clinical implications, Sports Med, 36, 929-939, 2006.

[2] E.C. Goddard, J.P. Dickey. Exercise acutely improves dynamic balance in individuals with unilateral knee osteoarthritis, International Journal of Human Movement and Sports Sciences, 7, 5-11, 2019.

[3] W. Lim. Easy method for measuring stretching intensities in real clinical settings and effects of different stretching intensities on flexibility, Journal of Back and Musculoskeletal Rehabilitation, 32, 579-585, 2019.

[4] J.B. Feland, H.N. Marin. Effect of submaximal contraction intensity in contract-relax proprioceptive neuromuscular facilitation stretching, Br J Sports Med, 38, E18, 2004.

[5] B. Khodayari, Y. Dehghani. The investigation of mid-term effect of different intensity of PNF stretching on improve hamstring flexibility, Procedia - Social and Behavioral Sciences, 46, 5741-5744, 2012.

[6] P.W. Sheard, T.J. Paine. Optimal contraction intensity during proprioceptive neuromuscular facilitation for maximal increase of range of motion, J Strength Cond Res, 24, 416-421, 2010. 
[7] P.W. Sheard, P.M. Smith, T.J. Paine. Athlete compliance to therapist requested contraction intensity during proprioceptive neuromuscular facilitation, Man Ther, 14, 539-543, 2009.

[8] T.M. Gomes, R. Simão, M.C. Marques, P.B. Costa, J. da Silva Novaes. Acute effects of two different stretching methods on local muscular endurance performance, J Strength Cond Res, 25, 745-752, 2011.

[9] L.E. Holt, T.W. Pelham, P.D. Campagna. Hemodynamics during a machine-aided flexibility protocol, Can J Appl Physiol, 20, 407-416, 1995.

[10] J.K. Petrella, J.-S. Kim, S.C. Tuggle, M.M. Bamman Contributions of force and velocity to improved power with progressive resistance training in young and older adults, Eur J Appl Physiol, 99, 343-351, 2007.

[11] C. Kisner, L.A. Colby, J. Borstad. Therapeutic Exercise: Foundations and Techniques, F.A. Davis, 2017.

[12] D.M. Pincivero, P.T. Dixon, A.J. Coelho. Knee extensor torque, work, and EMG during subjectively graded dynamic contractions, Muscle Nerve, 28, 54-61, 2003.

[13] S. Morishita, A. Tsubaki, M. Nakamura, S. Nashimoto, J.B. $\mathrm{Fu}, \mathrm{H}$. Onishi. Rating of perceived exertion on resistance training in elderly subjects, Expert Rev Cardiovasc Ther, 17, $135-142,2019$.

[14] B.B. Edin. Quantitative analyses of dynamic strain sensitivity in human skin mechanoreceptors, J Neurophysiol, $92,3233-3243,2004$

[15] B.B. Edin, A.B. Vallbo. Dynamic response of human muscle spindle afferents to stretch, J Neurophysiol, 63, 1297-1306, 1990.

[16] H. Johansson, P. Sjölander, P. Sojka. A sensory role for the cruciate ligaments, Clin Orthop Relat Res, 161-178, 1991.

[17] J.P. Roll, J.P. Vedel, E. Ribot. Alteration of proprioceptive messages induced by tendon vibration in man: a microneurographic study, Exp Brain Res, 76, 213-222, 1989.

[18] J.N. Bernier, D.H. Perrin. Effect of coordination training on proprioception of the functionally unstable ankle, J Orthop Sports Phys Ther, 27, 264-275, 1998.

[19] K. Kim, B. Choi, W. Lim. The efficacy of virtual reality assisted versus traditional rehabilitation intervention on individuals with functional ankle instability: a pilot randomized controlled trial, Disabil Rehabil Assist Technol, 14, 276-280, 2019.

[20] G. Lentell, B. Baas, D. Lopez, L. McGuire, M. Sarrels, P. Snyder. The contributions of proprioceptive deficits, muscle function, and anatomic laxity to functional instability of the ankle, J Orthop Sports Phys Ther, 21, 206-215, 1995.

[21] D.M. Pincivero, S.M. Thomas. The effects of knee joint angle and contractor intensity on perceived exertion, Percept Mot Skills, 125, 1123-1139, 2018.

[22] D. Toffin, J. McIntyre, J. Droulez, A. Kemeny, A. Berthoz. Perception and reproduction of force direction in the horizontal plane, J Neurophysiol, 90, 3040-3053, 2003.

[23] M.P. Kaufman, S.G. Hayes, C.M. Adreani, J.G. Pickar. Discharge properties of group III and IV muscle afferents, Adv Exp Med Biol, 508, 25-32, 2002.

[24] D.M. Rotto, M.P. Kaufman. Effect of metabolic products of muscular contraction on discharge of group III and IV afferents, J Appl Physiol, 64, 2306-2313, 1988.

[25] F. Thimm, K. Baum. Response of chemosensitive nerve fibers of group III and IV to metabolic changes in rat muscles, Pflugers Arch, 410, 143-152, 1987.

[26] B.L. Guthrie, J.D. Porter, D.L. Sparks. Corollary discharge provides accurate eye position information to the oculomotor system, Science, 221, 1193-1195, 1983.

[27] M. Kawato. Internal models for motor control and trajectory planning, Curr Opin Neurobiol, 9, 718-727, 1999.

[28] U. Proske, T. Allen. The neural basis of the senses of effort, force and heaviness, Exp Brain Res, 237, 589-599, 2019.

[29] J.F.A. Poulet, B. Hedwig. New insights into corollary discharges mediated by identified neural pathways, Trends Neurosci, 30, 14-21, 2007

[30] R.J. Robertson, B.J. Noble. Perception of physical exertion: methods, mediators, and applications, Exerc Sport Sci Rev, 25, 407-452, 1997

[31] A.W. Jackson, R.K. Dishman, S.B. Martin. Perceived leg extension and flexion forces of young adult men and women: comparison to previous findings, Res Q Exerc Sport, 73, 225-228, 2002.

[32] S. Kumar, M. Simmonds. The accuracy of magnitude production of submaximal precision and power grips and gross motor efforts, Ergonomics, 37, 1345-1353, 1994.

[33] B.L. Day, C.D. Marsden, J.A. Obeso, J.C. Rothwell Reciprocal inhibition between the muscles of the human forearm., J Physiol, 349, 519-534, 1984.

[34] R. Katz, A. Penicaud, A. Rossi. Reciprocal Ia inhibition between elbow flexors and extensors in the human., J Physiol, 437, 269-286, 1991.

[35] D.G. Watt, E.K. Stauffer, A. Taylor, R.M. Reinking, D.G. Stuart. Analysis of muscle receptor connections by spike-triggered averaging. 1. Spindle primary and tendon organ afferents, J Neurophysiol, 39, 1375-1392, 1976.

[36] D. Zytnicki, J. Lafleur, G. Horcholle-Bossavit, F. Lamy, L. Jami. Reduction of Ib autogenetic inhibition in motoneurons during contractions of an ankle extensor muscle in the cat, $\mathrm{J}$ Neurophysiol, 64, 1380-1389, 1990.

[37] W. Lim. Changes in pain following the different intensity of the stretching and types of physical stress, Physical Therapy Korea, 26, 63-69, 2019.

[38] W. Lim. Optimal intensity of PNF stretching: maintaining the efficacy of stretching while ensuring its safety, J Phys Ther Sci, 30, 1108-1111, 2018

[39] K.E. Light, S. Nuzik, W. Personius, A. Barstrom. Low-load prolonged stretch vs. high-load brief stretch in treating knee contractures, Phys Ther, 64, 330-333, 1984. 